\title{
Developing a Fuzzy Time Series Forecasting Model Based on Hedge Algebras and Particle Swarm Optimization
}

\author{
Nghiem Van Tinh*, Nguyen Tien Duy and Tran Thi Thanh \\ Faculty of Electronics, Thai Nguyen University of Technology, Thai Nguyen, Vietnam
}

('Corresponding author's e-mail: nghiemvantinh@tnut.edu.vn)

Received: 12 September 2020, Revised: 5 May 2021, Accepted: 12 May 2021

\begin{abstract}
In recent years, numerous fuzzy time series (FTS) forecasting models have been widely used. One of the important factors for obtaining high forecasting accuracy in fuzzy time series model is that the lengths of intervals in the universe of discourse. In this study, a hybrid forecasting model which uses hedge algebra (HA) and particle swarm optimization (PSO) is proposed to determine optimal lengths of intervals in FTS models. In that, HA is utilized as a tool to partition the universe of discourse into intervals with unequal-size corresponding to the semantic intervals calculated from the linguistic terms. After processing of generating the intervals, we define fuzzy sets based on the observation data of times series and use them to establish fuzzy relationship groups. Then, the proposed model is combined with the PSO technique to find the appropriate length of each interval with view to reaching the better forecasting accuracy rate. The performance of the proposed model is evaluated with the historical data of enrolments at the University of Alabama. The simulated results obtained indicate that the proposed model achieves higher forecasting accuracy compared other existing forecasting models and it can obtain better quality solutions for both the 1st-order and high-order FTS model.
\end{abstract}

Keywords: Fuzzy time series, Fuzzy relationship group, Hedge algebras, Particle swam optimization, Enrolments

\section{Introduction}

Dealing with the time series forecasting problem, many forecasting models have been introduced to advance the decision-making process concerning future, such as enrollments forecast for the next year, temperature prediction of the coming days, annual population forecasting, financial forecasting, ... Based on fuzzy set theory [1], Song and Chissom [2,3] proposed 2 FTS models by using max-min operations in fuzzy relationships to forecast the enrollments of the University of Alabama. Compared with the previous traditional forecasting models, such as regression analysis, moving average, autoregressive moving average and ARIMA model, the forecasting models in articles [2,3] can make better predictions with forecasting problems in which the historical data needs to be represented by linguistic values or uncertain data series. However, their models had many drawbacks such as huge computation when the fuzzy rule matrix is large and lack of persuasiveness in determining the universe of discourse and the length of intervals. Therefore, to avoid this shortcoming, Chen [4] developed a FTS forecasting model using simplified arithmetic operations rather than max-min composition operator in defuzzification process. In addition, research works in articles [5,6] pointed out the importance of assigning weights to resolve the issue of recurrent fuzzy relationship and to reflect the difference in their importance. From the expansion of the research [4] into a high-order fuzzy time series model [7] and the influence of the lengths of intervals in article [8] together with the development from the 1-factor FTS models into 2-factor FTS model [9] is the foundation for the strong development of FTS models in the next time periods. Recently, many authors have used different techniques in each stage of FTS model to improve forecasting accuracy. Chen and Tanuwijaya [10] used the automatic clustering method to partition the universe of discourse into different interval lengths in the fuzzification stage of the forecasting model. Some other researches combine optimization techniques with different FTS models to adjust and find the lengths of intervals from the universe of discourse [11-22]. Based on the idea of finding the suitable interval lengths, many models used clustering techniques to divide time series dataset into clusters, then adjust these clusters into intervals with different lengths such as: K-mean cluster [23,24] and clustered C-mean [25,26]. A completely dif- 
ferent way from fuzzy approach, information granules consider to be a sound option. Just recently, several related works have been presented. Ho et al. [27] has introduced a forecasting model based on the theory of HA [28] to apply for forecasting university enrolments. In which, the HA was used to model linguistic domains and variables instead of performing data fuzzification and defuzzification. In addition, Tung et al. [29] proposed a HA-based forecasting model to find different lengths of intervals in the universe of discourse by mapping the semantics of linguistic terms into fuzziness intervals. However, 2 these research works only focus on building a $1^{\text {st }}$-order forecasting model to forecasting the number of enrollments at the University of Alabama.

From analyzing of the research works above showed that the lengths of intervals and the order of fuzzy relationships are 2 critical factors for forecasting accuracy. Bearing in mind the idea in the using HA, in this paper we propose a hybrid fuzzy time series model combining HA and PSO for forecasting the enrollments of the University of Alabama [4]. In this research, HA is used to partition the universe of discourse into unequal-sized intervals by quantifying the linguistic terms themselves which is used to describe the historical values of fuzzy time series. After generating the intervals, the historical time series dataset is fuzzified based on the defined fuzzy sets. Each fuzzified time series value is then used to create the FLRs and divide them into groups. Later, all these fuzzy relationship groups are utilized to obtain the forecasting results based on the our defuzzification principle [30]. Finally, the proposed model is combined with the PSO algorithm to adjust the initial interval lengths for further increasing predictive accuracy.

\section{Materials and methods}

In this section, we briefly review basic concepts related to fuzzy time series [2,3], the HA [28] and PSO algorithm [31].

\section{Some basic definitions of FTS}

Based on the fuzzy set theory [1], Song and Chissom [2,3] introduced the definition of FTS and constructed its model by means of fuzzy relational equations, in which the values of historical time series data are presented by fuzzy sets. Let $U=\left\{\mathrm{u}_{1}, \mathrm{u}_{2}, \ldots, \mathrm{u}_{\mathrm{n}}\right\}$ be an universe of discourse; a fuzzy set $A$ of $U$ can be defined as $A=\left\{\mu_{\mathrm{A}}\left(\mathrm{u}_{1}\right) / \mathrm{u}_{1}+, \mu_{\mathrm{A}}\left(\mathrm{u}_{2}\right) / \mathrm{u}_{2} \ldots+\mu_{\mathrm{A}}\left(\mathrm{u}_{\mathrm{n}}\right) / \mathrm{u}_{\mathrm{n}}\right\}$, where $\mu_{\mathrm{A}}: \mathrm{U} \rightarrow[0,1]$ is the membership function of $A, \mu_{A}\left(u_{i}\right)$ indicates the degree of membership of $u_{i}$ in the fuzzy set $A, f_{A}\left(u_{i}\right) \in[0,1]$, and $1 \leq i \leq n$. The basic definitions related to FTS are summarized as below:

Definition 1: Fuzzy time series [2,3]

Let $Y(t)(t=\ldots, 0,1,2 \ldots)$, a subset of real numbers, be the universe of discourse on which the fuzzy sets $f_{i}(t)(i=1,2 \ldots)$ are defined in the universe of discourse $Y(t)$ and $F(t)$ is a collection of $f_{1}(t), f_{2}(t), \ldots$, then $\mathrm{F}(\mathrm{t})$ is called a FTS definition on $\mathrm{Y}(\mathrm{t})(\mathrm{t} \ldots, 0,1,2 \ldots)$.

Definition 2: Fuzzy logical relationship - FLR [2-4]

If there exists a fuzzy relationship $\mathrm{R}(\mathrm{t}-1, \mathrm{t})$, such that $\mathrm{F}(\mathrm{t})=\mathrm{F}(\mathrm{t}-1) * \mathrm{R}(\mathrm{t}-1, \mathrm{t})$, where "*" is an max-min composition operator, then $F(t)$ is said to be caused by $F(t-1)$. The relationship between $F(t)$ and $F(t-1)$ can be denoted by $F(t-1) \rightarrow F(t)$. Let $A_{i}=F(t)$ and $A_{j}=F(t-1)$, the relationship between $F(t)$ and $F(t-1)$ is denoted by fuzzy logical relationship $A_{i} \rightarrow A_{j}$ where $A_{i}$ and $A_{j}$ refer to the current state or the left-hand side and the next state or the right-hand side of fuzzy relationship.

Definition 3: $\lambda$-order fuzzy logical relationship [7]

Let $\mathrm{F}(\mathrm{t})$ be a fuzzy time series. If $\mathrm{F}(\mathrm{t})$ is caused by $\mathrm{F}(\mathrm{t}-1), \mathrm{F}(\mathrm{t}-2), \ldots, \mathrm{F}(\mathrm{t}-\lambda+1), \mathrm{F}(\mathrm{t}-\lambda)$ then this fuzzy relationship is represented by $\mathrm{F}(\mathrm{t}-\lambda), \ldots, \mathrm{F}(\mathrm{t}-2), \mathrm{F}(\mathrm{t}-1) \rightarrow \mathrm{F}(\mathrm{t})$ and is called an $\lambda$-order fuzzy time series.

Definition 4: Time-variant fuzzy relationship groups (TV-FRGs) [17]

The fuzzy logical relationship is defined by the relationship $F(t-1) \rightarrow F(t)$. If, let $F(t)=A_{i}(t)$ and $F(t-1)=A_{j}(t-1)$. The FLR between $F(t-1)$ and $F(t)$ can be denoted as $A_{j}(t-1) \rightarrow A_{i}(t)$. Also at the time $t$, we have the following fuzzy logical relationships: $A_{j}(t 1-1) \rightarrow A_{i 1}(t 1), \ldots, A_{j}(t \lambda-1) \rightarrow$ $A_{i \lambda}(t \lambda)$ with $t 1, t 2, \ldots, t \lambda \leq t$. It is noted that $A_{i}(t 1), A_{i}(t 1), \ldots$, and $A_{i}(t \lambda)$ with the same fuzzy set $A_{i}$ but appear at different times $t 1, t 2, \ldots$, and $t n$, respectively. It means that if these FLRs occur before $A_{j}(t-$ 1) $\rightarrow A_{i}(t)$, we can group these FLRs into a FRG according to the left-hand side of each FLR as $A_{j}(t-1) \rightarrow A_{i 1}(t 1), A_{i 2}(t 2), \ldots, A_{i \lambda}(t \lambda), A_{i}(t)$. It is named $1^{\text {st }}$ - order TV-FRGs. 


\section{Some basis concepts of hedge algebras [28]}

HA are created by Ho and Wechler [28] in 1990. In the field of time series forecasting, this theory is considered as a new approach to quantify the linguistic terms differing from the fuzzy set approach. In this study, the concepts of HA are employed as basis to partition the universe of discourse of time series into initial intervals with different lengths. Assume that there is a set of linguistic values of linguistic variable $\mathrm{X}$ which are sorted as follows: $X=\{$ Very Very low $<$ Very low $<$ low $<$ Little low $<$ Very Little low $<$ medium $<$ Very Little high $<$ Little big $<$ high $<$ Very high $<\cdots\}$. Each of linguistic variable $\mathcal{X}$ is represented by an algebraic structure as $\mathcal{A X}=(X, G, C, H, \leq)$ and called $\mathrm{HA}$, where $\mathrm{X}$ is the set of terms in $\mathcal{X} ; \leq$ denotes a natural semantically ordering relation on $\mathrm{X} ; \mathrm{G}=\left\{\mathrm{c}^{-}, \mathrm{c}^{+}\right\}, \mathrm{c}^{-} \leq \mathrm{c}^{+}$, is the set of primary generators, in which $\mathrm{c}^{+}$and $\mathrm{c}^{-}$are, respectively, the negative primary term and the positive one of a linguistic variable $\mathrm{X}, \mathrm{C}=\{0,1, \mathrm{w}\}$ a set of constants, with $\left(\mathbf{0} \leq \mathrm{c}^{-} \leq \mathbf{W} \leq \mathrm{c}^{+} \leq \mathbf{1}\right)$; $\mathrm{H}=\mathrm{H}^{-} \cup \mathrm{H}^{+}$, với $\mathrm{H}^{-}=\left\{\mathrm{h}_{-\mathrm{q}} \geq \cdots \geq \mathrm{h}_{-2} \geq \mathrm{h}_{-1}\right\}$ is the set of all negative hedges of $\mathrm{X}, \forall \mathrm{h} \in \mathrm{H}^{-}$then $\mathrm{hc}^{+} \leq \mathrm{c}^{+}$and $\mathrm{H}^{+}=\left\{\mathrm{h}_{1} \leq \mathrm{h}_{2} \leq \cdots \leq \mathrm{h}_{\mathrm{p}}\right\}$ is the set of all positive ones of $\mathrm{X}, \forall \mathrm{h} \in \mathrm{H}^{+}$then $\mathrm{hc}^{+} \geq \mathrm{c}^{+}$. Example $\mathrm{H}^{-}=\{$Little $>$Rather $\}, \mathrm{H}^{+}=\{$More $<$Very $\} . \forall \mathrm{x} \in \mathrm{X}, \mathrm{x}=\mathrm{h}_{\mathrm{n}} \mathrm{h}_{\mathrm{n}-1} \ldots \mathrm{h}_{1} \mathrm{c}, \mathrm{h}_{\mathrm{j}} \in \mathrm{H}$ with $\mathrm{c} \in \mathrm{G}$. If $\mathrm{X}$ and $\mathrm{H}$ are linearly ordered sets, then $\mathcal{A X}=(\mathrm{X}, \mathrm{G}, \mathrm{C}, \mathrm{H}, \leq)$ is called linear $\mathrm{HA}$, furthermore, if $\mathrm{AX}$ is equipped with additional operations $\sum$ and $\Phi$ that are, respectively, infimum and supremum of $\mathrm{H}(\mathrm{x})$, then it is called complete linear hedge algebras (ClinHA) and denoted $\mathcal{A X}=(\mathrm{X}, \mathrm{G}, \mathrm{C}, \mathrm{H}, \Sigma, \Phi \leq)$ [32]. Some general definitions of HA are given as follows:

Definition 5: Let $A X=(X, G, C, H, \leq)$ be a ClinHA. $f m: X \rightarrow[0,1]$ is said to be a fuzziness measure of terms in $\mathrm{X}$ if:

1) $f m\left(c^{-}\right)+f m\left(c^{+}\right)=1$ and $\sum_{h \in H} f m(h x)=f m(x)$, with $\forall x \in X$.

2) For the constants $\mathbf{0}, \mathbf{W}$ and $\mathbf{1}, \mathrm{fm}(\mathbf{0})=\mathrm{fm}(\mathbf{W})=\mathrm{fm}(\mathbf{1})=0$.

3) For $\forall x, y \in X, \forall h \in H, \frac{f m(h x)}{f m(x)}=\frac{f m(h y)}{f m(y)}$, that is this proportion does not depend on specific elements and, therefore, it is called fuzziness measure of the hedge $h$ and denoted by $\mu(h)$. The properties of $\mathrm{fm}(\mathrm{x})$ and $\mu(\mathrm{h})$ are introduced as follows:

Proposition 1: Let fm is the fuzziness measure function on $\mathrm{X}$, the following statements hold.

With $\in X, x=h_{n} h_{n-1} \ldots h_{1} c, h_{j} \in H, c \in G$

1) $\mathrm{fm}(\mathrm{hx})=\mu(\mathrm{h}) \mathrm{fm}(\mathrm{x}), \forall \mathrm{x} \in \mathrm{X}$.

2) $\sum_{-q<i<p, i \neq 0} f m\left(h_{i} c\right)=f m(c)$.

3) $\sum_{-q<i<p, i \neq 0} f m\left(h_{i} x\right)=f m(x)$.

4) $f m(x)=f m\left(h_{n} h_{n-1} \ldots h_{1} c\right)=\mu\left(h_{n}\right) \mu\left(h_{n-1}\right) \ldots \mu\left(h_{1}\right) f m(c)$.

5) $\sum_{i=-1}^{-q} \mu\left(h_{i}\right)=\alpha$ and $\sum_{i=1}^{p} \mu\left(h_{i}\right)=\beta$, with $\alpha, \beta>0$ and $\alpha+\beta=1$.

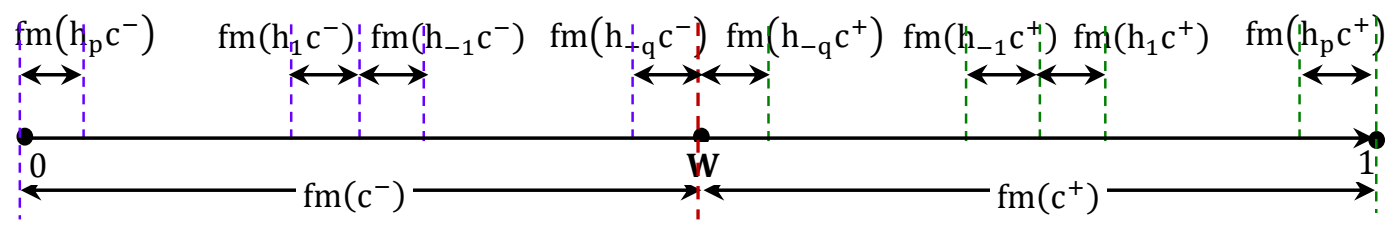

Figure 1 The order of elements $x \in X, h_{j} \in H, c \in G$.

Definition 6: The fuzziness interval of the linguistic terms $x \in X$, denoted by $\Im(x)$, is a subinterval of $[0,1]$, if $|\mathfrak{I}(x)|=f m(x)$ where $|\mathfrak{I}(x)|$ is the length of $f m(x)$, and recursively determined by the length of $\mathrm{x}$ as follows:

1) If length of $x$ is equal to $1(1(x)=1)$, that mean $x \in\left\{c^{-}, c^{+}\right\}$, then $\left|\mathfrak{S}\left(\mathrm{c}^{-}\right)\right|=f \mathrm{fm}\left(\mathrm{c}^{-}\right),\left|\mathfrak{S}\left(\mathrm{c}^{+}\right)\right|=\mathrm{fm}\left(\mathrm{c}^{+}\right)$ and $\mathfrak{I}\left(\mathbf{c}^{-}\right) \leq \mathfrak{I}\left(\mathrm{c}^{+}\right)$;

2) Suppose that $n$ is the length of $x(1(x)=n)$ and fuzziness interval $\mathfrak{S}(x)$ has been defined with $|\mathfrak{I}(x)|$ $=f m(x)$. The set $\left\{\mathfrak{J}\left(h_{j} x\right) \mid j \in\left[-q^{\wedge} p\right]\right\}$, where $\left[-q^{\wedge} p\right]=\{j \mid-q \leq j \leq-1$ or $1 \leq j \leq p\}$, is a partition of $\mathfrak{J}(x)$ and we have: for $\operatorname{Sgn}\left(\mathrm{h}_{\mathrm{p}} \mathrm{x}\right)=-1, \mathfrak{I}\left(\mathrm{h}_{\mathrm{p}} \mathrm{x}\right) \leq \mathfrak{I}\left(\mathrm{h}_{\mathrm{p}-1} \mathrm{x}\right) \leq \ldots \leq \mathfrak{I}\left(\mathrm{h}_{1} \mathrm{x}\right) \leq \mathfrak{I}\left(\mathrm{h}_{1} \mathrm{x}\right) \leq \ldots \leq \mathfrak{I}\left(\mathrm{h}_{-\mathrm{q}} \mathrm{x}\right)$; for $\operatorname{Sgn}\left(\mathrm{h}_{\mathrm{p}} \mathrm{x}\right)=+1$, $\mathfrak{I}\left(\mathrm{h}_{-\mathrm{q}} \mathrm{x}\right) \leq \mathfrak{I}\left(\mathrm{h}_{-\mathrm{q}+1} \mathrm{x}\right) \leq \ldots \leq \mathfrak{I}\left(\mathrm{h}_{-1} \mathrm{x}\right) \leq \mathfrak{J}\left(\mathrm{h}_{1} \mathrm{x}\right) \leq \ldots \leq \mathfrak{I}\left(\mathrm{h}_{\mathrm{p}} \mathrm{x}\right)$. 


\section{Particle swarm optimization algorithm}

PSO algorithm had been proposed by Kannedy and Eberhart [31] in 1965. It is considered as a tool to resolve with optimization problems, where a set of potential solutions is represented by a swarm of particles and each particle is move through the search space (d-dimensional space) for search the optimal solution. When particles moving, all particles (i.e, $\mathrm{N}$ particles) have fitness values which are evaluated by fitness function and the position of the best particle among all particles found so far is kept and each particle keeps its personal best position which has passed previously. In the movement process of particles, each $\mathrm{k}^{\text {th }}(1 \leq \mathrm{k} \leq \mathrm{N})$ particle associated with the velocity vector $\mathrm{V}_{\mathrm{ki}}=\left[v_{k, 1}, v_{k, 2}, \ldots, v_{k, d}\right]$ and the position vector $\mathrm{X}_{\mathrm{ki}}=\left[x_{k, 1}, x_{k, 2}, \ldots, x_{k, d}\right]$ of particle are updated by the best position $P_{b e s t \_k d}=\left[p_{k, 1}, p_{k, 2}, \ldots, p_{k, d}\right]$ encountered by the particle so far and the best position global $\mathrm{G}_{\mathrm{best}}=\min \left(P_{\text {best_kd }}^{t}\right)$ found by the overall best out of all the particles in the population. The briefly summarizes steps of the standard PSO algorithm in Algorithm 1 as follows:

\section{Algorithm 1 The standard PSO algorithm}

Step 1: Initialize random positions $x_{k i}$; random velocities $v_{k i}$ in $d$ dimensional space $(i=1,2, \ldots, d)$;

- Positions of each particle are randomly determined and saved in a vector $X_{\mathrm{kd}}$ as follows:

$\mathrm{X}_{\mathrm{ki}}=\left[x_{k, 1}, x_{k, 2}, \ldots, x_{k, d}\right]$

Where; $x_{\mathrm{ki}}$ denotes $\mathrm{i}^{\text {th }}$ position of $\mathrm{k}^{\text {th }}$ particle. $\mathrm{N}$ is the number of particles in a swarm;

- Velocities are randomly determined and stored in a vector $\mathrm{V}_{\mathrm{kd}}$ given below:

$\mathrm{V}_{\mathrm{ki}}=\left[v_{k, 1}, v_{k, 2}, \ldots, v_{k, d}\right]$

Step 2: According to the fitness function value $\mathrm{f}(\mathrm{x})$, the values of $P_{\text {best_kd }}$ and $\mathrm{G}_{\text {best }}$ of particles given in Eq. (3) are determined as: $\mathrm{P}_{\text {best_kd }}=\left[\mathrm{p}_{\mathrm{k}, 1}, \mathrm{p}_{\mathrm{k}, 2}, \ldots, \mathrm{p}_{\mathrm{k}, \mathrm{d}}\right]$.

Where, $P_{\text {best } k \text { kd }}$ is a vector stores the positions corresponding to the $\mathrm{k}^{\text {th }}$ particle's best individual performance and calculated as:

$P_{\text {best_kd }}^{t+1} f(x)=\left\{\begin{array}{c}P_{\text {best }}^{t+1}, \text { if } f\left(x_{k i}^{t+1}\right)>P_{\text {best_ki }}^{t} \\ f\left(x_{k i}^{t+1}\right), \text { if } f\left(x_{k i}^{t+1}\right) \leq P_{\text {best_ki }}^{t}\end{array}\right.$

and $\mathrm{G}_{\text {best }}=\operatorname{minimum}\left(P_{\text {best } \_k i}\right)$ denotes the best one of all personal best positions of all particles within the swarm.

Step 3: $c_{1}$ and $c_{2}$ are 2 learning factors which control the influence of the social and cognitive components $\left(c_{1}=c_{2}=2\right)$, respectively. $\omega$ is the time-varying inertia weight.

In each iteration $t$, the parameter $\omega$ is calculated as follows:

$\omega^{t}=\omega_{\max }-\frac{t *\left(\omega_{\max }-\omega_{\min }\right)}{i t e r_{-} \max }$

Where, iter_max denotes the maximum iteration number.

Step 4: The new velocity and position of each particle can be updated by using the current velocity and distance from the $P_{\text {best }}$ to $G_{\text {best }}$ as shown in Eqs. (4) and (5), respectively.

$$
\begin{aligned}
& \mathrm{V}_{\mathrm{ki}}^{\mathrm{t}+1}=\omega^{\mathrm{t}} * \mathrm{~V}_{\mathrm{ki}}^{\mathrm{t}}+\mathrm{c}_{1} * \mathrm{R} 1() *\left(\mathrm{P}_{\text {best_kd }}-\mathrm{X}_{\mathrm{ki}}^{\mathrm{t}}\right)+\mathrm{c}_{2} * \mathrm{R} 2() *\left(\mathrm{G}_{\mathrm{best}}-\mathrm{X}_{\mathrm{ki}}^{\mathrm{t}}\right) \\
& \mathrm{X}_{\mathrm{ki}}^{\mathrm{t}+1}=\mathrm{X}_{\mathrm{ki}}^{\mathrm{t}}+\mathrm{V}_{\mathrm{ki}}^{\mathrm{t}+1}
\end{aligned}
$$

Where, R1 and R2 are generated random values in the domain $[0,1]$. 
Step 5: Steps 2 to 4 are repeated until a predetermined maximum iteration number (iter_max) is reached.

\section{A hybrid FTS forecasting model based on HA and PSO}

This section, we introduce a novel FTS forecasting model which is combined between HA and PSO for improving forecasted accuracy. The framework of proposed model is presented in Figure 2, including 3 stages; the $1^{\text {st }}$ stage is to partition the historical data into initial intervals based on HA; the $2^{\text {nd }}$ stage is to establish the FTS model and create forecasting rules and the final stage is to find optimal lengths of intervals by applying PSO algorithm. To implement these stages, all historical enrollments data [4] are utilized for illustrating forecasting process. This dataset has been selected to forecast with the great amount of research works which have been published in the literatures [3,4,6-9,10-12,14,15,17,21-24,27,29,30]. The 3 stages of proposed model are present as follows.

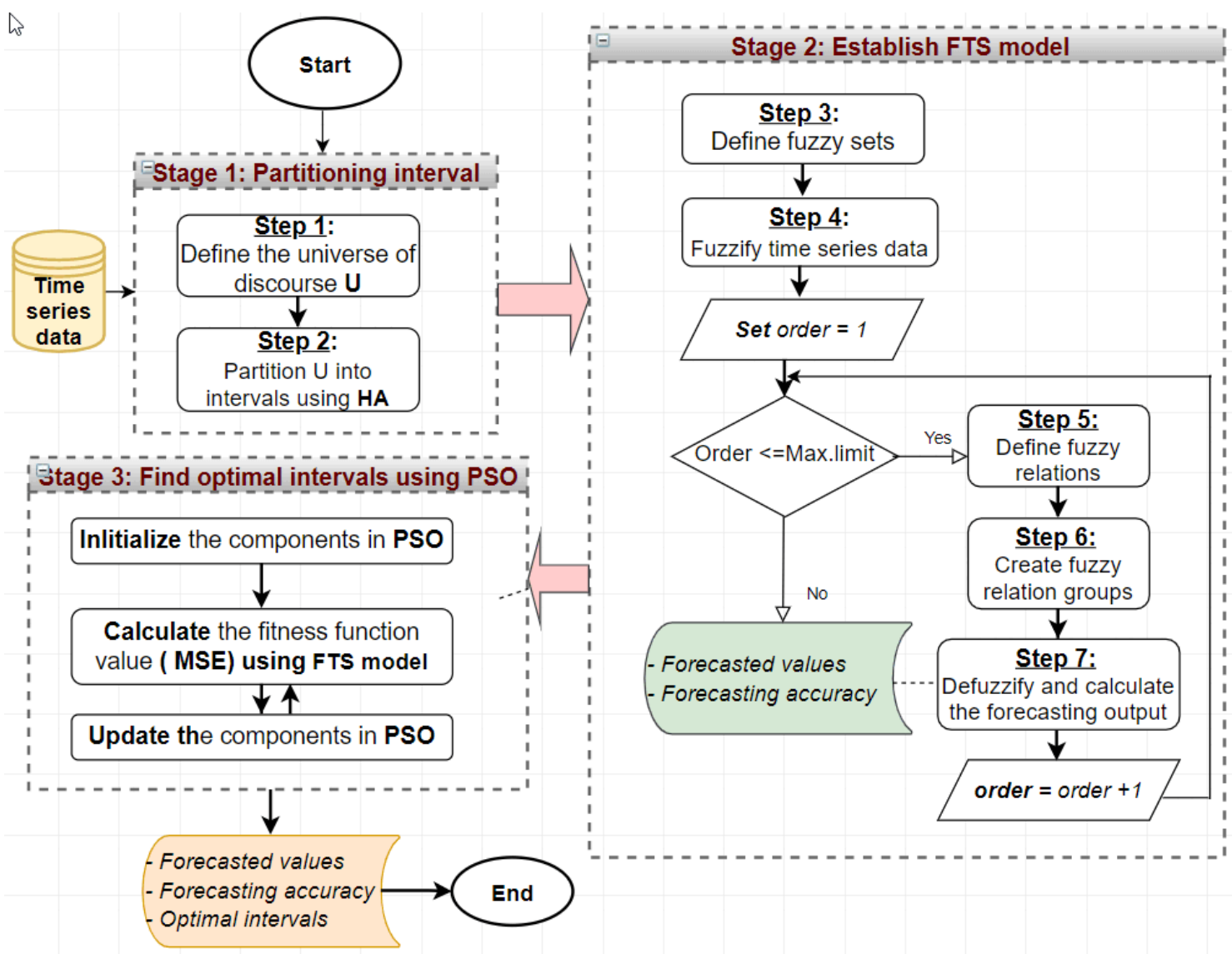

Figure 2 flowchart of the proposed FTS forecasting model.

\section{Stages 1 and 2: Constructing forecasting model based on FTS and HA}

In this section, a forecasting model based on combining the FTS and HA for forecasting enrolments of the University. Initially, the HA is applied to divide the universe of discourse into initial intervals with unequal-lengths by quantitative mapping of linguistic terms into fuzzy intervals. Based on these newly obtained intervals, we defined fuzzy sets and fuzzy historical data on each divided interval. From these fuzzified values, we derive the FLRs and establish fuzzy relationship groups according to [17]. Later, all these FRGs are used to obtain the forecasting results based on the defuzzification method [30]. The proposed forecasting model can be given step-by-step as follows:

Step 1: Define the universe of discourse $U$ of historical time series data Let $U=\left[D_{\min }-D_{1}, D_{\max }+D_{2}\right]$ is universe of discourse. To define $U$, the minimum value $D_{\min }$ and the maximum value $\mathrm{D}_{\max }$ of the historical time series data is defined. In order to ensure the forecasting values bounded in the universe of discourse $U 2$ positive integers $D_{1}$ and $D_{2}$ are properly selected, re- 
spectively. From historical enrolments time series, $U$ is defined as $U=[13000,20000]$, where $D_{\min }$, = $13055, D_{\max }=19337, D_{1}=55, D_{2}=663, \mathrm{LU}=7000$.

Step 2: Partition $\mathrm{U}$ into different intervals based on HA

This step uses HA with structure as $\mathcal{A X}=(X, G, C, H, \leq)$, where $\mathrm{X}$ is the set of terms of the linguistic variable "enrollments" $\{X=\operatorname{dom}($ enrollments $)\} ; \leq$ denotes a natural semantically ordering relation on $\mathrm{X} ; G=\left\{c^{-}, c^{+}\right\}=\{$Low, High $\}$, Low $(\mathrm{Lw}) \leq$ High $(\mathrm{Hi}) ; \mathrm{C}=\{0, \mathrm{w}, 1\}$ a set of constants, with $\left(0 \leq c^{-} \leq\right.$ $\left.W \leq c^{+} \leq 1\right)$ and $\mathrm{H}=\{$ Very, Little $\}$. To compare the forecasted results of the proposed model with other models. In this paper, we use the number of intervals equal to 7 and 14 which are the number of linguistic terms used to quantify the time series values. In particular, suppose that the linguistic terms are given in Table 1. Based on these linguistic terms, we define the corresponding intervals of them in domain $\mathrm{U}$.

Table 1 The number of language terms.

\begin{tabular}{|c|c|}
\hline $\begin{array}{c}\text { Number of } \\
\text { linguistic terms }\end{array}$ & Linguistic terms and its order \\
\hline 7 & $\begin{array}{c}\mathrm{A}_{1}=\text { Very Very Low }\left(\text { VVLw) }<\mathrm{A}_{2}=\text { Little Verry Low }(\mathrm{LVLw})<\mathrm{A}_{3}=\text { Little Little }\right. \\
\text { Low (LLLw) }<\mathrm{A}_{4}=\text { Very Little Low }(\mathrm{VLLw})<\mathrm{A}_{5}=\text { Very Little High }(\mathrm{VLHi})< \\
\mathrm{A}_{6}=\text { Little Little High }\left(\text { LHHi) }<\mathrm{A}_{7}=\text { Very High }(\mathrm{VHi})\right. \\
\mathrm{A}_{1}=\text { VVLw }<\mathrm{A}_{2}=\text { LLVLw }<\mathrm{A}_{3}=\text { VLVLw }<\mathrm{A}_{4}=\text { VLLLw }<\mathrm{A}_{5}=\text { LLLLw }< \\
\mathrm{A}_{6}=\text { LVLLw }<\mathrm{A}_{7}=\text { VVLLw }<\mathrm{A}_{8}=\text { VVLHi }<\mathrm{A}_{9}=\text { LVLHi }<\mathrm{A}_{10}=\text { LLLHi }< \\
\mathrm{A}_{11}=\text { VLLHi }<\mathrm{A}_{12}=\text { VLVHi }<\mathrm{A}_{13}=\text { LLVHi }<\mathrm{A}_{14}=\text { VVHi }\end{array}$ \\
\hline
\end{tabular}

Step 2.1: The domain $U=[13000,20000]$ is mapped to the domain $[0,1]$

Suppose the value of 16807 in the time series dataset is the average value, then we can set up the following parameters: $\mathrm{fm}(\mathrm{Low})=\frac{16807-13000}{20000-13000}=0.544, \mathrm{fm}($ High $)=1-0.544=0.456$ and $\mathrm{LU}=20000-$ $13000=7000$. Mapping these values to $\mathrm{U}$, we have $\operatorname{covfm}(\mathrm{Low})$ and $\operatorname{covfm}($ High) that are determined, respectively as $\mathrm{fm}(\mathrm{Low}) \times \mathrm{LU}=0.544 \times 7000=3808, \mathrm{fm}($ high $) \times \mathrm{LU}=0.456 \times 7000=3192$. In this paper, we can choose $\mu($ Little $)=0.48, \mu($ Very $)=1-0.48=0.52$. Based on $\mu($ Little $), \mu($ Very $)$ value, the value of $\alpha, \beta$ is defined as $0.48,0.52$, respectively.

From here, the fuzziness interval of linguistic terms in the domain [0,1] can be calculated: $\mathrm{fm}(\mathrm{VVLw})=0.1471, \mathrm{fm}(\mathrm{LVLw})=0.1358, \mathrm{fm}(\mathrm{LLLw})=0.1253, \mathrm{fm}(\mathrm{VLLw})=0.1358, \mathrm{fm}(\mathrm{VLHi})=$ $0.11138, \mathrm{fm}(\mathrm{LLHi})=0.1051, \mathrm{fm}(\mathrm{VHi})=0.2371$.

Step 2.2: Define the fuzzy interval of linguistic variable in the universe of discourse Based on Step 2.1, the linguistic values of terms belong to fuzziness interval is calculated as follows: $\operatorname{covfm}\left(\mathrm{A}_{1}\right)=\mu($ Verry $) \times \mu($ Very $) \times \operatorname{covfm}($ Low $)=0.52 \times 0.52 \times 3808=1029.683$; $\operatorname{covfm}\left(\mathrm{A}_{2}\right)=\mu($ Little $) \times \mu($ Very $) \times \operatorname{covfm}($ Low $)=0.48 \times 0.52 \times 3808=950.477$; $\operatorname{covfm}\left(\mathrm{A}_{3}\right)=\mu($ Little $) \times \mu($ Little $) \times \operatorname{covfm}($ Low $)=0.48 \times 0.48 \times 3808=479.36$; $\operatorname{covfm}\left(\mathrm{A}_{4}\right)=\mu($ Very $) \times \mu($ Little $) \times \operatorname{covfm}($ Low $)=0.52 \times 0.48 \times 3808=950.477$;

$\operatorname{covfm}\left(A_{7}\right)=\mu($ Very $) \times \operatorname{covfm}($ High $)=0.52 \times 3192=1659.84$

Mapping the value of linguistic terms to the domain of the universe of discourse $U$, we get the intervals corresponding to linguistic terms, which are listed as below:

For 7 linguistic terms, obtaining 7 intervals as $\mathrm{u}_{1}=[13000,14029.68), \mathrm{u}_{2}=[14029.68,14980.2)$, $\mathrm{u}_{3}=[14980.2,15,857.5), \mathrm{u}_{4}=[15857.5,16808), \mathrm{u}_{5}=[16808,17605), \mathrm{u}_{6}=[17605,18340.16), \mathrm{u}_{7}=$ [18340.16, 20000].

For 14 linguistic terms, obtaining 14 intervals as $\mathrm{u}_{1}=[13000,13539.5), \mathrm{u}_{2}=[13539.5,14079), \mathrm{u}_{3}$ $=[14079,14438.5), \mathrm{u}_{4}=[14438.5,14798), \mathrm{u}_{5}=[14798,15157.5), \mathrm{u}_{6}=[15157.5,15517), \mathrm{u}_{7}=[15517$, $15756.5), u_{8}=[15756.5,15996), u_{9}=[15996,16316.5), u_{10}=[16316.5,16637), u_{11}=[16637,17117.5)$, $\mathrm{u}_{12}=[17117.5,17598), \mathrm{u}_{13}=[17598,18799), \mathrm{u}_{14}=[18799,20000]$. 
Step 3: Define linguistic terms $A_{i}$ which represented by fuzzy sets

Each of interval in Step 2 represents a linguistic value of linguistic variable "enrolments". For 7 intervals, there are 7 linguistic values to represent different regions in the universe of discourse on U. Each linguistic value represents a fuzzy set $A_{i}$ and its definitions is described in Eqs. (7) and (8) as follows:

$$
\begin{aligned}
& \mathrm{A}_{\mathrm{i}}=a_{i 1} / \mathrm{u}_{1}+a_{i 2} / \mathrm{u}_{2}+\ldots+a_{i j} / \mathrm{u}_{j}+\cdots+a_{i 7} / \mathrm{u}_{7} \\
& a_{i j}=\left\{\begin{array}{cr}
1 & j=i \\
0.5 & j=i-1 \text { or } j=i+1 \\
0 & \text { otherwise }
\end{array}\right.
\end{aligned}
$$

Here, the symbol ' + ' denotes the set union operator, $a_{i j} \in[0,1](1 \leq i \leq 7,1 \leq j \leq 7)$, $u_{j}$ is the ${ }^{j}$ th interval of the universe of discourse. The value of $a_{i j}$ indicates the grade of membership of $u_{j}$ in the fuzzy set $A_{i}$. For simplicity, the different membership values of fuzzy set $A_{i}$ are selected by according to triangular membership function and which is defined Eq. (8). From Eqs. (7) and (8), a fuzzy set contains 7 intervals. Contrarily, each of interval belongs to all fuzzy sets with different membership degrees. Therefore, based on the obtained intervals from enrollments dataset, the corresponding linguistic values are illustrated in Figure 3.

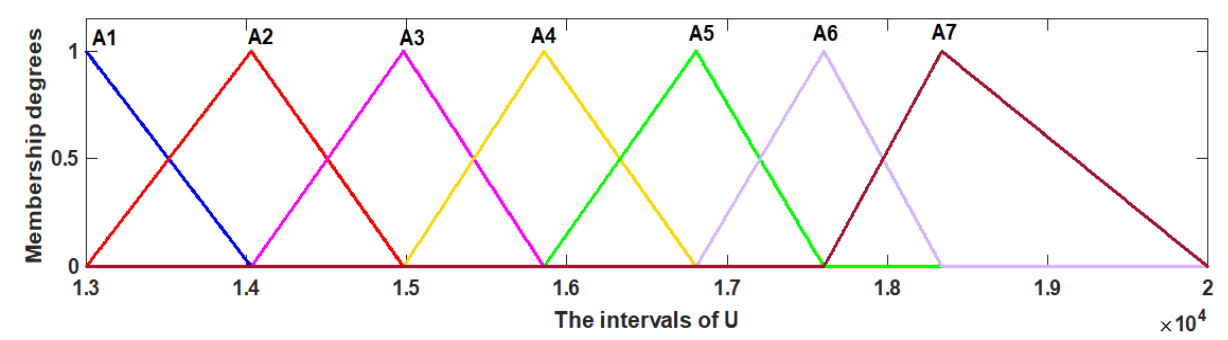

Figure 3 The fuzzy sets are defined by intervals using the triangular membership function.

Step 4: Fuzzy the historical time series data

To fuzzify the historical time series data, it is essential to obtain the degree of membership value of each data value belonging to each $\mathrm{u}_{i}$ for each year. If the maximum membership value of 1 day's observation occurs at $\mathrm{u}_{i}$, and $(1 \leq \mathrm{i} \leq 7)$, then the fuzzified value for that particular year is considered as $A_{i}$. For example, the historical enrolment of year 1972 is 13563 , and it belongs to interval $\mathrm{u}_{1}$ because 13563 is within $[13000,14078.56)$. So, we then assign the linguistic value "Very Very Low" (e.g., the fuzzy set $A_{1}$ ) corresponding to interval $u_{1}$ to it. Considering 2 time series data $Y(t)$ and $F(t)$ at year $t$, where $Y(t)$ is actual data and $F(t)$ is the fuzzy set of $Y(t)$. According to Eq. (7), the fuzzy set $A_{1}$ has the maximum membership value at the interval $u_{1}$. Therefore, the historical data time series on date $Y(1972)$ is fuzzified to $A_{1}$. The completed fuzzified results of the enrolments data are presented in Table 2.

Table 2 Fuzzified historical enrollments data of the University of Alabama.

\begin{tabular}{cccc}
\hline Year & Real data & Fuzzy sets & Linguistic values \\
\hline 1971 & 13055 & $\mathrm{~A}_{1}$ & Very Very Low \\
1972 & 13563 & $\mathrm{~A}_{1}$ & Very Very Low \\
--- & --- & --- & ----------- \\
1991 & 19337 & $\mathrm{~A}_{7}$ & Little Little High \\
1992 & 18876 & $\mathrm{~A}_{7}$ & Little Little High \\
\hline
\end{tabular}


Step 5: Create all $\lambda$-order fuzzy logical relationships $(\lambda \geq 1)$.

Based on Definitions 3 and 4 , to establish a $\lambda$-order fuzzy logical relationship, we should find out any relationship which has the $\mathrm{F}(\mathrm{t}-\lambda), \mathrm{F}(\mathrm{t}-\lambda+1), \ldots, \mathrm{F}(\mathrm{t}-1) \rightarrow \mathrm{F}(\mathrm{t})$, where $\mathrm{F}(\mathrm{t}-\lambda), \mathrm{F}(\mathrm{t}-\lambda+$ $1), \ldots, F(t-1)$ and $F(t)$ are called the current state and the next state, respectively. Then a $\lambda$-order fuzzy relationship in the training phase is got by replacing the corresponding linguistic values. For example, supposed $\lambda=1$ from Table 2, a fuzzy relation $A_{1} \rightarrow A_{1}$ is got as $F(1971) \rightarrow F(1972)$. So on, we get the $1^{\text {st }}$-order fuzzy relationships are shown in column 4 of Table 3, where there are 22 fuzzy relationships; the first 21 relations are called the trained patterns, and the last one is called the untrained pattern (in the testing phase). For the untrained pattern, relation 22 has the fuzzy relation $A_{7} \rightarrow \#$ as it is created by the relation $\mathrm{F}(1992) \rightarrow \mathrm{F}(1993)$, since the linguistic value of $\mathrm{F}(1993)$ is unknown within the historical data, and this unknown next state is denoted by the symbol ' $\#$ '. The same way, suppose $\lambda=2$, a fuzzy relationship $\left(A_{1}, A_{1}\right) \rightarrow A_{1}$ is got as $(F(1971), F(1972)) \rightarrow F(1973)$. The complete 2-order fuzzy relationships are listed in column 5 of Table 3 .

Table 3 The complete the $1^{\text {st }}$-order and the $2^{\text {nd }}$-order FLRs.

\begin{tabular}{ccccc}
\hline No & Year & Fuzzy set & $\mathbf{1}^{\text {st }}$-order FLRs & 2 $^{\text {nd }}$-order FLRs \\
\hline & 1971 & $\mathrm{~A}_{1}$ & & \\
1 & 1972 & $\mathrm{~A}_{1}$ & $\mathrm{~A}_{1} \rightarrow \mathrm{A}_{1}$ & \\
2 & 1973 & $\mathrm{~A}_{1}$ & $\mathrm{~A}_{1} \rightarrow \mathrm{A}_{1}$ & $\mathrm{~A}_{1}, \mathrm{~A}_{1} \rightarrow \mathrm{A} 1$ \\
3 & 1974 & $\mathrm{~A}_{2}$ & $\mathrm{~A} 1 \rightarrow \mathrm{A}_{2}$ & $\mathrm{~A}_{1}, \mathrm{~A}_{1} \rightarrow \mathrm{A}_{2}$ \\
--- & --- & --- & -- & -- \\
21 & 1992 & $\mathrm{~A} 7$ & $\mathrm{~A}_{7} \rightarrow \mathrm{A}_{7}$ & $\mathrm{~A}_{7}, \mathrm{~A}_{7} \rightarrow \mathrm{A}_{7}$ \\
22 & 1993 & $\#$ & $\mathrm{~A}_{7} \rightarrow \#$ & $\mathrm{~A}_{7}, \mathrm{~A}_{7} \rightarrow \#$ \\
\hline
\end{tabular}

Step 6: Construct all fuzzy relationship groups (FRGs)

In this step, an approach is different from the approach in articles [4,10-14] in the way where the fuzzy relationship groups are created. In previous approaches [4,5], the recurrent FLRs were simply ignored when fuzzy relationship groups were established or these repeated fuzzy relationships is mentioned, but it is not suitable at each of forecasting time, respectively. In this study, we rely on a concept of time-variant fuzzy logical relationship group [17] which presented in Definition 5 to create FRGs, called TV-FRGs. To explain this, assume that $\lambda=1$. We consider the $31^{\text {st }}$-order FLRs at 3 different times $t=1972,1973$, 1974 in column 4 of Table 3 as follows: $F(t=1972): A_{1} \rightarrow A_{1} ; F(t=1973): A_{1} \rightarrow A_{1} ; F(t=1974): A_{1}$ $\rightarrow A_{2}$; all of them with the same fuzzy set $A_{1}$ on the left - hand side. Then, if considering at forecasting time $t=1992$, we have obtained a $1^{\text {st }}$-order FRG (i.e., No 1 ) as follows: $A_{1} \rightarrow A_{1}$. With forecasting time $t=1993$, before that there 2 FLRs with the same on left-hand side, these FLRs can be grouped into a FRG as No 2: $A_{1} \rightarrow A_{1}, A_{1}$. With forecasting time $t$ as 1994, then the group G3 is expressed as follows $A_{1} \rightarrow$ $A_{1}, A_{1}, A_{2}$. The similar explanation for $\lambda=2$, we complete the all $1^{\text {st }}$-order and $2^{\text {nd }}$-order TV-FRGs and shown in column 2 and column 3 of Table 4 , respectively.

Table 4 The complete the $1^{\text {st }}$-order and $2^{\text {nd }}$-order TV-FRGs.

\begin{tabular}{ccc}
\hline No & $\mathbf{1}^{\text {st }}$-order TV-FRGs & 2 $^{\text {nd }}$-order TV-FRGs \\
\hline 1 & $A_{1} \rightarrow A_{1}$ & $\left(A_{1}, A_{1}\right) \rightarrow A_{1}$ \\
2 & $A_{1} \rightarrow A_{1}, A_{1}$ & $\left(A_{1}, A_{1}\right) \rightarrow A_{1}, A_{2}$ \\
3 & $A_{1} \rightarrow A_{1}, A_{1}, A_{2}$ & $\left(A_{1}, A_{2}\right) \rightarrow A_{3}$ \\
-- & --- & --- \\
20 & $A_{7} \rightarrow A_{7}, A_{7}$ & $\left(A_{7}, A_{7}\right) \rightarrow A_{7}, A_{7}$ \\
21 & $A_{7} \rightarrow A_{7}, A_{7}, A_{7}$ & $\left(A_{7}, A_{7}\right) \rightarrow \#$ \\
22 & $A_{7} \rightarrow \#$ & \\
\hline
\end{tabular}


Step 7: Defuzzify and calculate the forecasting output values

The last step of the proposed model is to defuzzify the forecasting state to a crisp output value from fuzzy forecasting rules. In particular, to defuzzify the fuzzified data values, the our defuzzified principle in article [30] is presented to compute the forecasted value for all $1^{\text {st }}$-order and high-order time variant FRGs in training phase. Next, we use a defuzzified principle [14] for computing with the unknown linguistic value in testing phase. The forecasting principles is presented as follows:

Principle 1: Apply to calculate forecasting output value in the training phase

To calculate forecasting value output based on information of the each group. We divide each corresponding interval with respect to the fuzzy sets in the next state of the TV-FRGs into 3 sub-intervals with the same length. The forecasted value for each group is defined as follows:

Forecasted_value $=\frac{1}{2 * n} \sum_{i=1}^{n}\left(s u b m_{i k}+\right.$ Value_lu $\left._{i k}\right)$

Where, $\mathrm{n}$ denotes the total number of fuzzy sets on the next state of TV-FRG.

$\checkmark$ subm $_{i k}$ denotes the midpoint value of one of 3 sub-intervals $(1 \leq k \leq 3)$ with respect to i-th linguistic value in the next state of FRG that the real data at forecasting time falls into this subinterval.

$\checkmark \quad$ Value_l $_{i k}$ is one of 2 values belongs to the lower bound and upper bound value of one of 3 sub-intervals which has the real data at forecasting time falls within sub-interval $u_{\mathrm{ik}}$ (i.e., $u_{\mathrm{ik}}=$ $\left.\left[L_{i k}, U_{i k}\right]\right)$.

- If the real data value at forecasting time minors the midpoint value of sub-interval $u_{\mathrm{ik}}$, then Value_l $u_{i k}$ is assigned as the lower bound of sub-interval $u_{\mathrm{ik}}$; else Value_lu $u_{i k}$ is assigned as the upper bound of sub-interval $u_{\mathrm{ik}}$.

For example, suppose that we want to calculate the forecasting value in year 1972. Based on column 4 of Table 5 shown that the $2^{\text {nd }}$-order fuzzy relationship group $\mathrm{G} 1\left(\mathrm{~A}_{1} \rightarrow \mathrm{A}_{1}\right)$ is formed from a FLR with next state $A_{1}$ appearing at year 1972, where the highest membership degree of $A_{1}$ fall into interval $u_{1}=$ $[13000,14029.68)$. Thus, we partition the interval $u_{1}$ into 3 sub-intervals which are $u_{1.1}=$ $[13000,13343.23), u_{1.2}=[13343.23,13686.45)$ and $u_{1.3}=[13686.45,14029.68)$, respectively. In addition, the historical data of year 1972 with respect to linguistic value $A_{1}$ of 13563 and it fall within sub-interval $u_{1,2}=[13343.23,13686.45)$. The The corresponding mid-value for the sub-interval $u_{1.2}$ is $s u b m_{1.2}$ (13514.84). Following, the value of Value $l u_{i k}$ obtained by comparing between the real value of year 1972 and the midpoint value of sub-interval $u_{1.2}$. By this way, the value of $V a l_{-} l u_{i k}\left(V_{a l} l u_{21}\right)$ is assigned equal to 13686.45 . Finally, the forecasting output value of year 1972 can be computed according to Eq. (10) as follows:

Forecasted_value $=\frac{1}{2}(13514.84+13686.45)=13600.65$

By the same way, we can get the forecasting value for the $2^{\text {nd }}$-order fuzzy relationship group $\left(\left(A_{1}, A_{1}\right) \rightarrow\right.$ $\mathrm{A}_{1}$ ) appearing at year 1973 as 13943.9.

Principle 2: Using for calculating forecasting output value in the testing phase

For testing phase, we calculate forecasted value for a group of fuzzy relationship which has the unidentified linguistic value on the right-hand side based on the master vote scheme [14]. Assume there a $\lambda$-order fuzzy relationship group as $A_{t-\lambda}, A_{t-(\lambda+1)}, A_{t 1} \rightarrow \#$, the forecasting value is estimated according to Eq. (11), where the symbol $w_{h}$ is the highest votes predefined by user for each other problem, $\lambda$ is the order of the FLRs, the symbols $M_{t-1}, M_{t-2} \ldots$ and $M_{t-\lambda}$ are the middle values of the corresponding intervals which related to the latest fuzzy set and other fuzzy sets on the left-hand side of fuzzy relationship group having the maximum membership values of $A_{t-1}, A_{t-2}, \ldots$, and $A_{t-\lambda}$ occur at intervals $u_{t 1}, u_{t 2}, \ldots$, and $\mathrm{u}_{\mathrm{t}-\lambda}$, respectively.

Forecated_value $=\frac{\left(M_{t-1} * w_{h}\right)+M_{t-2}+\cdots+M_{t-\lambda}}{w_{h}+(\lambda-1)}$ 
Based on the 2 forecasting principles above and fuzzy relationship groups in Table 4, we complete forecasting results for the enrolments the period from 1971 to 1992 based on $1^{\text {st }}$-order and high-order time variant FRGs under 7 intervals are shown in column 4 and 5 of Table 5, respectively.

Table 5 The complete forecasted outputs based on the $1^{\text {st }}$-order and $2^{\text {nd }}$-order FTS.

\begin{tabular}{ccccc}
\hline Year & Actual data & Fuzzy set & $\begin{array}{c}\mathbf{1}^{\text {st }} \text {-order forecasted } \\
\text { value }\end{array}$ & $\begin{array}{c}\mathbf{2}^{\text {nd }} \text {-order forecasted } \\
\text { value }\end{array}$ \\
\hline 1971 & 13055 & A1 & --- & -- \\
1972 & 13563 & A1 & 13600.6 & --- \\
1973 & 13867 & A1 & 13772.3 & 13943.9 \\
1974 & 14696 & A2 & 14095.7 & 14343.2 \\
--- & --- & --- & --- & -- \\
1991 & 19337 & A7 & 19308.4 & 19308.4 \\
1992 & 18876 & A7 & 19124 & 19031.8 \\
MSE & & & $\mathbf{1 2 9 6 2 3 . 3 4}$ & $\mathbf{7 0 1 8 8 . 3 7}$ \\
\hline
\end{tabular}

The efficiency of the proposed forecasting model is evaluated using various statistical indexes, namely Mean Square Error (MSE) and Mean Absolute Percentage Error (RMSE). The lower value of MSE, RMSE indicates better performance of proposed model. The evaluation criterions are determined by the following equations:

$$
\begin{aligned}
& \text { MSE }=\frac{1}{n} \sum_{i=\lambda}^{\mathrm{n}}\left(\mathrm{F}_{\mathrm{i}}-\mathrm{R}_{\mathrm{i}}\right)^{2} \\
& \text { RMSE }=\sqrt{\frac{1}{\mathrm{n}} \sum_{\mathrm{i}=\lambda}^{\mathrm{n}}\left(\mathrm{F}_{\mathrm{i}}-\mathrm{R}_{\mathrm{i}}\right)^{2}}
\end{aligned}
$$

Where, $R_{i}$ and $F_{i}$ note the actual and forecasted value at time $\mathrm{i}$, respectively, $\mathrm{n}$ is the total number of years to be forecasted, $\lambda$ is the order of fuzzy logical relationship.

\section{Stage 3: A hybrid FTS forecasting model based on combining the HA and PSO}

In this section, we present the hybrid FTS forecasting model by combining HA and PSO algorithm with the aim to improve forecasting accuracy. In which, PSO algorithm is used to minimize the MSE value by adjusting the lengths of the initial intervals which are determined by HA and membership values, respectively. The proposed forecasting model is named FTSHA-PSO. The briefly explanation of the FTSHA-PSO are given as following. In the FTSHA-PSO model, for the training phase, each particle is used to represent the partitioning of time series data (e.g., $\mathrm{n}$ intervals). Assume that the lower bound and upper bound of the universe of discourse $\mathrm{U}$ be $x_{0}$ and $x_{n}$, respectively. Each particle denotes a vector containing of $n-1$ elements as $x_{1}, x_{2}, \ldots, x_{n-2}$ and $x_{n-1}$, where $(1 \leq i \leq n-1)$ and $x_{i} \leq x_{i+1}$. From these $\mathrm{n}-1$ elements, define the $\mathrm{n}$ intervals as $u_{1}=\left[x_{0}, x_{1}\right], u_{2}=\left[x_{1}, x_{2}\right], \ldots, u_{i}=\left[x_{i-1}, x_{i}\right], \ldots$ and $u_{n}=$ $\left[x_{n-1}, x_{n}\right]$, respectively. In case of movement of particle in a swarm from 1 position to another position, the elements of the corresponding new array always require to be adjusted in an ascending order such that $x_{1} \leq x_{2} \leq \cdots \leq x_{n-1}$. In processing for the training phase, the FTSHA-PSO model permits each particle moving form current position to other position by Eqs. (5) and (6), and repeats the steps until the stopping criterion is satisfied. If the stopping criterion is satisfied, then all the FRGs obtained by the global best position $\left(G_{\text {best }}\right)$ among all personal best positions $\left(P_{\text {best }}\right)$ of all particles which used to forecast the new testing data in testing phase. Here, the MSE value in Eq. (12) is used to evaluate the forecasted accuracy of each particle. The complete steps of the proposed model are presented in Algorithm 2 as follows: 
1 Input: Historical time series data

2 Output: The forecasting results and the MSE value $(\mathrm{MSE}=\mathrm{Gbest}=\min ($ Pbest $)$ )

Begin

3 Define the initial intervals by applying HA and use forecasting steps in Subsection above to reach the initial forecasting accuracy (MSE).

4. Initialize: a population of $\mathrm{N}$ particles

$\checkmark \quad$ The initial position $\mathrm{X}_{\mathrm{ki}}$ and the velocity $\mathrm{V}_{\mathrm{ki}}$ of all particles, respectively.

$\checkmark$ The initial personal best position vectors of the ${ }^{k}$ th particle is the same as its initial position vector at the beginning and find Gbest

5. do

5.1. for each particle $\mathrm{k},(1 \leq \mathrm{k} \leq \mathrm{N})$ do

$\checkmark$ Following the steps in the part above sequentially, from step 3 to step 7 such as: defining linguistic terms, fuzzify all historical, determining all $\lambda$ - order fuzzy logical relationships, establishing all $\lambda$ - order TV-FRGs, defuzzify forecasting values, calculating the MSE value for each particle

$\checkmark$ The new Pbest of all particles is saved according to the MSE values.

end for

5.2. The new Gbest of all particles is saved according to the MSE values

6 . for each particle $\mathrm{k},(1 \leq \mathrm{k} \leq \mathrm{N})$ do

$\checkmark \quad$ The particle $\mathrm{k}$ is moved to another position according to Eqs. (5) and (6)

end for

$\checkmark \quad$ Update $\omega$ according to Eq. (4)

while (the maximum moving steps(iter_max) or the minimum MSE are reached)

End.

Algorithm 3 The FTSHA-PSO algorithm in the testing phase

The proper lengths of intervals and order of FLRs obtained in Algorithm 2 that are used to estimate untrained data in the testing phase based on the Principle 2 in the proposed model.

Example: The illustrating of the FTSHA-PSO model in the training phase is presented as follows. In this example, let the number of intervals and particles be 7 and 4 respectively, and the FTSHA-PSO model uses the PSO to obtain all the $2^{\text {nd }}$-order FLRs by adjusting the length of intervals for the historical enrolments data. In proposed FTS model, we have the universe of discourse on $U=[13000,20000]$, where lower bound $\mathrm{x}_{0}=13000$ and upper bound $\mathrm{x}_{7}=20000$, respectively. For finding the optimal solution, we define values for the parameters used in Eqs. (5) and (6) as: The range of $\mathrm{x}_{k i}$ be limited to $(13000,20000]$, the range of $\mathrm{v}_{k i}$ be limited to $[-100,100]$, the values of $C_{1}$ and $C_{2}$ be 2 , and the value of $\omega=0.9$ (where $\omega$ linearly decreases its value to the lower bound, 0.4 , through the whole training process) and maximum number of iterations be 2 , respectively. The positions and velocities of all particles are initialized randomly and listed in Tables 6 and 7, respectively.

In Table 6, we have shown the 7 intervals for each particle which are $u_{1}=\left[x_{0}, x_{1}\right], u_{2}=$ $\left[x_{1}, x_{2}\right], \ldots, u_{i}=\left[x_{i-1}, x_{i}\right], \ldots$ and $u_{n}=\left[x_{n-1}, x_{n}\right]$, respectively. Where, the intervals of the initial position of particle 1 are established as the same the one which are created from HA in Subsection 3.1 and listed as $\mathrm{u}_{1}=[13000,14029.68), \mathrm{u}_{2}=[14029.68,14980.2), \mathrm{u}_{3}=[14980.2,15857.5), \mathrm{u}_{4}=[15857.5,16808)$, $\mathrm{u}_{5}=[16808,17605), \mathrm{u}_{6}=[17605,18340.16), \mathrm{u}_{7}=[18340.16,20000]$. Next, we follow the steps of Algorithm 2 and achieve the optimal intervals which are utilized for obtaining the forecasting results. The MSE value of particle1 is calculated based on Eq. (12). The MSE values for the remaining 3 particles are found in a similar manner. Based on the corresponding MSE value, every particle updates its own personal best positions (Pbest) so far. For simplicity, the initial Pbests are considered for the initial positions of all particles. The Pbests of all particles so far are shown in Table 8. From Table 8, the global best position Gbest $=\min ($ Pbest $)$ is created by particle 3, because its MSE value is the least among all particles so far. After the $1^{\text {st }}$ iteration, all particles move to the $2^{\text {nd }}$ positions according to Eqs. (5) and (6). The $2^{\text {nd }}$ positions and the corresponding new MSE values of all particles are presented in Table 9.

Table 6 Randomly generated initial positions of all particles. 


\begin{tabular}{cccccccc}
\hline Particle & $\boldsymbol{x}_{\mathbf{1}}$ & $\boldsymbol{x}_{\mathbf{2}}$ & $\boldsymbol{x}_{\mathbf{3}}$ & $\boldsymbol{x}_{\mathbf{4}}$ & $\boldsymbol{x}_{\mathbf{5}}$ & $\boldsymbol{x}_{\mathbf{6}}$ & MSE \\
\hline 1 & $\mathbf{1 4 0 2 9 . 6 8}$ & $\mathbf{1 4 9 8 0 . 2}$ & $\mathbf{1 5 8 5 7 . 5}$ & $\mathbf{1 6 8 0 8}$ & $\mathbf{1 7 6 0 5}$ & $\mathbf{1 8 3 4 0 . 1 6}$ & $\mathbf{7 0 1 8 5 . 7 3}$ \\
2 & 13485.34 & 14156.19 & 14217.29 & 18109.05 & 18305.1 & 19046.01 & 426939.77 \\
3 & 13572.29 & 14395.55 & 15206.81 & 15572.54 & 16668.41 & 17504.68 & 31861.41 \\
4 & 14368.55 & 15098.79 & 15672.91 & 16495.91 & 17598.12 & 18025.22 & 55020.22 \\
\hline
\end{tabular}

Table 7 Randomly generated initial velocities of all particles.

\begin{tabular}{ccccccc}
\hline Particle & $\boldsymbol{v}_{\mathbf{1}}$ & $\boldsymbol{v}_{\mathbf{2}}$ & $\boldsymbol{v}_{\mathbf{3}}$ & $\boldsymbol{v}_{\mathbf{4}}$ & $\boldsymbol{v}_{\mathbf{5}}$ & $\boldsymbol{v}_{\mathbf{6}}$ \\
\hline 1 & -4.45 & -75.51 & -40.73 & -20.91 & 39.05 & -93.04 \\
2 & 13.48 & -92.09 & 76.32 & 86.76 & 52.71 & -58.5 \\
3 & -82.46 & 14.13 & -45.76 & 55.36 & 46.77 & -38.01 \\
4 & -31.41 & -60.12 & -26.92 & -75.18 & 77.02 & 3.83 \\
\hline
\end{tabular}

Table 8 The initial Pbest of all particles; the global best position is created by particle 3 as its MSE is the least among 4 particles.

\begin{tabular}{cccccccc}
\hline Particle & $\boldsymbol{x}_{\mathbf{1}}$ & $\boldsymbol{x}_{\mathbf{2}}$ & $\boldsymbol{x}_{\mathbf{3}}$ & $\boldsymbol{x}_{\mathbf{4}}$ & $\boldsymbol{x}_{\mathbf{5}}$ & $\boldsymbol{x}_{\mathbf{6}}$ & MSE \\
\hline 1 & 14029.68 & 14980.2 & 15857.5 & 16808 & 17605 & 18340.16 & 70185.73 \\
2 & 13485.34 & 14156.19 & 14217.29 & 18109.05 & 18305.1 & 19046.01 & 426939.77 \\
3 & 13572.29 & 14395.55 & 15206.81 & 15572.54 & 16668.41 & 17504.68 & 31861.41 \\
4 & 14368.55 & 15098.79 & 15672.91 & 16495.91 & 17598.12 & 18025.22 & 55020.22 \\
\hline
\end{tabular}

Table 9 The $2^{\text {nd }}$ positions of all particles.

\begin{tabular}{cccccccc}
\hline Particle & $\boldsymbol{x}_{\mathbf{1}}$ & $\boldsymbol{x}_{\mathbf{2}}$ & $\boldsymbol{x}_{\mathbf{3}}$ & $\boldsymbol{x}_{\mathbf{4}}$ & $\boldsymbol{x}_{\mathbf{5}}$ & $\boldsymbol{x}_{\mathbf{6}}$ & MSE \\
\hline 1 & 13929.68 & 14880.2 & 15757.5 & 16708 & 17505 & 18240.16 & 54313.57 \\
2 & 13565.06 & 14256.19 & 14317.29 & 18009.05 & 18205.1 & 18946.01 & 402776.58 \\
3 & 13498.08 & 14408.27 & 15165.63 & 15622.36 & 16710.5 & 17470.47 & 36398.23 \\
4 & 14268.55 & 14998.79 & 15572.91 & 16395.91 & 17498.12 & 17925.22 & 70457.58 \\
\hline
\end{tabular}

By comparing the MSE values shown in Table 8 with those listed in Table 9, it is obvious that particle 1 and particle 2 in Table 9 attained a better position than their own Pbest values so far. Thus, the 2 particles update their own Pbest values, which are shown in Table 10. The new Gbest is obtained by particle 3, because its MSE value is the least among all the particles so far. The FTSHA-PSO model is accomplished by repeating the above steps until the maximum number of iterations is reached. Finally, the proper lengths of intervals are achieved by the Gbest value that the particle 3 attains so far, and are employed for obtaining the final forecasting. These obtained results used to forecast the new testing data in the testing phase based on Algorithm 3.

Table 10 The $2^{\text {nd }}$ Pbest of all particles and the Gbest value is obtained by particle 3 .

\begin{tabular}{cccccccc}
\hline Particle & $\boldsymbol{x}_{\mathbf{1}}$ & $\boldsymbol{x}_{\mathbf{2}}$ & $\boldsymbol{x}_{\mathbf{3}}$ & $\boldsymbol{x}_{\mathbf{4}}$ & $\boldsymbol{x}_{\mathbf{5}}$ & $\boldsymbol{x}_{\mathbf{6}}$ & MSE \\
\hline 1 & 13929.68 & 14880.2 & 15757.5 & 16708 & 17505 & 18240.16 & 54313.57 \\
2 & 13565.06 & 14256.19 & 14317.29 & 18009.05 & 18205.1 & 18946.01 & 402776.58 \\
3 & 13572.29 & 14395.55 & 15206.81 & 15572.54 & 16668.41 & 17504.68 & $\mathbf{3 1 8 6 1 . 4 1}$ \\
4 & 14368.55 & 15098.79 & 15672.91 & 16495.91 & 17598.12 & 18025.22 & 55020.22 \\
\hline
\end{tabular}

\section{Results and discussion}


In this paper, the proposed model is applied to forecast enrollments of University of Alabama [4]. The forecasting results of proposed model are compared with those of corresponding models in the literature for various order and different intervals. For implementing the experiment, we use visual studio 2019 environment with C\# programming language on an Intel Core i7 PC with 8GB RAM. Based on parameters in Table 11, the FTSHA-PSO model is executed 20 times on enrolments dataset with various number of orders and intervals. Then, the best result of all runs is recorded to be the final forecast result. All forecasted accuracies are evaluated by MSE and RMSE value which are presented in Eqs. (12) and (13), respectively.

Table 11 The parameters of PSO used in the FTSHA-PSO model for forecasting enrolments.

\begin{tabular}{cc}
\hline The parameters in PSO & Values of parameters \\
\hline The number of particles $\mathrm{N}=$ & 50 \\
The max number of iterations iter_max $=$ & 200 \\
The value of inertial weight $\omega$ is decreased by & $\omega_{\max }=0.9$ to $\omega_{\min }=0.4$ \\
The coefficient $\mathrm{C} 1=\mathrm{C} 2=$ & 2 \\
The position in search range: $\mathbf{X}=$ & {$[13000,20000]$} \\
The velocity in search range: $\mathbf{V}=$ & {$[-100,100]$} \\
\hline
\end{tabular}

\section{Forecasting results based on the $\mathbf{1}^{\text {st }}$-order fuzzy time series}

In order to evaluate the effectiveness of the proposed model based on the $1^{\text {st }}$-order FTS with the number of intervals equal to 7, the forecasting models is introduced in articles [33-36,27,29] were considered for comparison. From the parameters are set for the enrolments data. A comparison in term of RMSE value between the FTSHA-PSO model and its counterparts are shown in Table 12. Based on forecasting results in Table 12, the FTSHA-PSO model gets the smallest RMSE value of $\mathbf{1 7 2 . 9}$ among all the compared models. Differences between the FTSHA-PSO model and models mentioned above is the way which the fuzzy relationship group and method of partitioning the universe of discourse are applied to establish the forecasting model. Three forecasting models in works [33-35] are constructed based on Chen's model to forecast different problems and apply information granules to partition, while the FTSHA-PSO model uses HA for determining unequal-sized interval lengths. In addition, 2 models in articles [27,29] based on HA and Chen's fuzzy relationship groups to structure the forecasting model, while the FTSHA-PSO model uses an approach that benefits from the concept of time-variant FRG [17] to establish the forecasting model. Finally, the FTSHA-PSO is different from the model [36] in the partitioning the universe of discourse they used, the former uses the HA which combines with PSO for finding the optimal interval lengths but latter utilizes the maximum spanning tree based fuzzy clustering algorithm for partitioning intervals with different lengths in the intuitionistic FTS forecasting model.

Table 12 A comparison of the forecasting results of the FTSHA-PSO model with its counterparts based on $1^{\text {st }}$-order FTS under 7 intervals.

\begin{tabular}{ccccccccc}
\hline Year & Real data & {$[\mathbf{3 3}]$} & {$[\mathbf{3 4}]$} & {$[\mathbf{3 5}]$} & {$[\mathbf{2 7}]$} & {$[\mathbf{3 6}]$} & {$[\mathbf{2 9}]$} & FTSHA-PSO \\
\hline 1972 & 13563 & 13486 & 13944 & 14279 & 13820 & 13500 & 13865 & 13619.24 \\
1973 & 13867 & 14156 & 13944 & 14279 & 13820 & 14155 & 14082 & 13729.16 \\
--- & --- & --- & --- & -- & --- & --- & -- & -- \\
1991 & 19337 & 18808 & 18933 & 19257 & 19135 & 19575 & 19165 & 19321.66 \\
1992 & 18876 & 18808 & 18933 & 19257 & 19135 & 18855 & 15219 & 19167.49 \\
RMSE & & $\mathbf{5 7 8 . 3}$ & $\mathbf{5 0 6}$ & $\mathbf{4 4 5 . 2}$ & $\mathbf{4 4 1 . 3}$ & $\mathbf{3 5 0 . 9}$ & $\mathbf{2 1 0 . 9}$ & $\mathbf{1 7 2 . 9}$ \\
\hline
\end{tabular}

In addition, the FTSHA-PSO model is also executed 20 runs to be compared with various $1^{\text {st }}$-order FTS models under number of intervals of 14 intervals. Five forecasting models are presented in research 
works $[4,6,11,14,15,17,35]$ were selected for comparison. A comparison of the forecasted results is shown in Table 13 where the number of intervals is 14 for all forecasting models. At the same intervals, it is obvious that the FTSHA-PSO model has the MSE value 5123 which is the lowest among all forecasting models compared.

Table 13 A comparison of the forecasting results of the FTSHA-PSO model with its counterparts based on $1^{\text {st }}$-order FTS under number of intervals of 14.

\begin{tabular}{cccccccccc}
\hline Year & real data & {$[4]$} & {$[\mathbf{6}]$} & {$[\mathbf{1 1}]$} & {$[\mathbf{1 4}]$} & {$[\mathbf{1 5}]$} & {$[\mathbf{1 7 ]}$} & {$[\mathbf{3 5}]$} & FTSHA-PSO \\
\hline 1971 & 13055 & & & & & & & & \\
1972 & 13563 & 14000 & 13653 & 13714 & 13555 & 13579 & 13434 & 13512 & 13558.12 \\
1973 & 13867 & 14000 & 13653 & 13714 & 13994 & 13812 & 13841 & 13998 & 13863.28 \\
---- & --- & --- & ---- & ---- & ---- & --- & --- & ---- & --- \\
1991 & 19337 & 19000 & 19059 & 19149 & 19340 & 19260 & 19340 & 19666 & 19262.03 \\
1992 & 18876 & 19000 & 19059 & 19014 & 19014 & 19031 & 18820 & 18718 & 19035.97 \\
MSE & & $\mathbf{4 0 7 5 0 7}$ & $\mathbf{3 1 6 8 4}$ & $\mathbf{3 5 3 2 4}$ & $\mathbf{2 2 9 6 5}$ & $\mathbf{8 2 2 4}$ & $\mathbf{7 4 7 5}$ & $\mathbf{1 4 5 3 4}$ & $\mathbf{6 6 6 5 . 8 9}$ \\
RMSE & & $\mathbf{6 3 8 . 4}$ & $\mathbf{1 7 8}$ & $\mathbf{1 8 7 . 9}$ & $\mathbf{1 5 1 . 5}$ & $\mathbf{9 0 . 7}$ & $\mathbf{8 6 . 5}$ & $\mathbf{1 2 0 . 6}$ & $\mathbf{8 1 . 6}$ \\
\hline
\end{tabular}

\section{Forecasting results based on the high-order fuzzy time series}

In this subsection, all historical enrolments dataset [4] covering a period from year 1971 to 1992 are partitioned into 2 parts to implement comparison results of the FTSHA-PSO with the ones of the existing methods, based on various high-orders. The $1^{\text {st }}$ part including 19 observations from year 1971 to 1989 is used as training data set and the $2^{\text {nd }}$ part consists of 3 observations is used as the testing data set. The performance of the FTSHA-PSO and the compared models are evaluated using the MSE and RMSE function.

\section{Experimental results in the training phase}

The FTSHA-PSO model is evaluated through the different high-order FLRs of fuzzy time series. In particular, in order to verify the superiority in the forecasted accuracy of the FTSHA-PSO model with number of intervals equal to 7 , the accuracies from cited papers in $[7,12,14,15,17]$ are selected for comparing. A comparison of the forecasting results is listed in Table 14, in what the number of intervals is established for all forecasting models equal to 7. At the same intervals 7, the FTSHA-PSO model gets the lowest MSE values which are 12457.8, 529.54, 443.47, 412.39, 366.42, 286.26, 163.27 and 371.13 for $2^{\text {nd }}-$ order, $3^{\text {rd }}$-order, $4^{\text {th }}$-order, $5^{\text {th }}$-order, $6^{\text {th }}$-order, $7^{\text {th }}$-order, $8^{\text {th }}$-order and $9^{\text {th }}$-order fuzzy time series, respectively. It can be seen that the FTSHA-PSO model achieves more precise than any other existing models under different high-order fuzzy relationships at all. Among all fuzzy relationships is done in the model, the FTSHA-PSO model obtains the lowest MSE value of 14420.4 with $8^{\text {th }}$-order fuzzy relationships. The major difference between FTSHA-PSO model and the compared models is in establishing fuzzy relationship groups and optimization technique they used. In optimization method, the model [12] performs genetic algorithm but the models in articles [14,15,17] and the FTSHA-PSO model proceed the PSO algorithm to achieve the best intervals, respectively. In addition to using PSO to find suitable intervals, the FTSHA-PSO model incorporates HA to partition the different initial intervals of the Universe of discourse instead of equal length intervals. In the determining of fuzzy relationship groups, the FTSHAPSO model is constructed from model [17], the remaining models in articles [7,12,14,15] are designed based on Chen's structure [4]. From the above analysis, it can be seen that the FTSHA-PSO model gives more convincing forecasting results compared to its counterparts based on the high-order FTS.

Table 14 A comparison of the results obtained between the FTSHA-PSO model and its counterparts based on the various high-order FTS with 7 intervals. 


\begin{tabular}{ccccccc}
\hline Order & {$[7]$} & {$[\mathbf{1 2}]$} & {$[\mathbf{1 4}]$} & {$[\mathbf{1 5}]$} & {$[\mathbf{1 7 ]}$} & FTSHA-PSO \\
\hline 2 & 89093 & 67834 & 67123 & 19594 & 19868 & 12457.8 \\
3 & 86694 & 31123 & 31644 & 31189 & 31307 & 529.54 \\
4 & 89376 & 32009 & 23271 & 20155 & 23288 & 443.47 \\
5 & 94539 & 24984 & 23534 & 20366 & 23552 & 412.39 \\
6 & 98215 & 26980 & 23671 & 22276 & 23684 & 366.42 \\
7 & 104056 & 26969 & 20651 & 18482 & 20669 & 286.26 \\
8 & 102179 & 22387 & 17106 & 14778 & 17116 & $\mathbf{1 6 3 . 2 7}$ \\
9 & 102789 & 18734 & 17971 & 15251 & 17987 & 371.13 \\
Average MSE & $\mathbf{9 5 8 6 7 . 6 3}$ & $\mathbf{3 1 3 7 7 . 5}$ & $\mathbf{2 8 1 2 1 . 3 8}$ & $\mathbf{2 0 2 6 1 . 3 8}$ & $\mathbf{2 2 1 8 3}$ & $\mathbf{1 8 7 8 . 7 9}$ \\
\hline
\end{tabular}

In addition, the FTSHA-PSO model is also compared with its counterparts which are introduced in papers $[7,12,14,15,37]$ based on the different high-order fuzzy time series with number of intervals of 14 . From the parameters are expressed in Table 6. The FTSHA-PSO model is executed 20 runs, and the best result of runs is taken to be the final result. A comparison of the forecasting accuracy with various highorders and the same number of intervals between the FTSHA-PSO model and its counterparts are listed in Table 15. Where, the FTSHA-PSO model is different from the model [37] in the way that the method of constructing forecasted model they used. The former applies the concept of the time-variant fuzzy relationship group but the latter proceeds the adaptive time-variant fuzzy time series to establish the forecasting model, respectively. In addition, the forecasting model in article [7] and the FTSHA-PSO model, both of them use the $5^{\text {th }}$-order fuzzy relationship but our FTSHA-PSO model is much more superior in term of forecasting accuracy. Remaining forecasting models in articles $[12,14,15]$, they use the fuzzy logical relationship with number of orders is larger, but the results obtained from our model are also better than the existing competing models. In particular, from Table 15, it is obvious that our forecasting model gets the MSE value of $\mathbf{1 8}$ which is the smallest among all compared forecasting models. This can conclude that the proposed model provides the superior forecasting performance compared to its counterparts based on the various high-order FLRs at all.

Table 15 A comparison of the forecasting results obtained between the FTSHA-PSO model and its counterparts based on the various high-order FTS with 14 intervals.

\begin{tabular}{cccccccc}
\hline Years & Real data & {$[7]$} & {$[37]$} & {$[\mathbf{1 2}]$} & {$[\mathbf{1 4}]$} & {$[\mathbf{1 5}]$} & FTSHA-PSO \\
\hline 1971 & 13055 & & & & & & \\
1972 & 13563 & & & & & & \\
1973 & 13867 & & 14934.5 & & & & \\
1974 & 14696 & & 15590 & & & & 15314 \\
1975 & 15460 & & 15422.9 & & & & 15608 \\
1976 & 15311 & 15500 & 15603 & & & & 15858 \\
1977 & 15603 & 15500 & 15861 & & & & 16803 \\
1978 & 15861 & 15500 & 16807 & & & & 16920 \\
1979 & 16807 & 16500 & 16919 & 16846 & & & 16390 \\
1980 & 16919 & 16500 & 16388 & 16846 & 16890 & 16920 & -- \\
1981 & 16388 & 16500 & 15553.9 & 16420 & 16395 & 16388 & - \\
--- & --- & ----- & 19332 \\
1991 & 19337 & 19500 & 18876 & 19334 & 19337 & 19335 & 18876 \\
1992 & 18876 & 18500 & 14934.5 & 18910 & 18882 & 18882 & $\mathbf{1 8}$ \\
MSE & & $\mathbf{8 6 6 9 4}$ & $\mathbf{5 3 0 8 4}$ & $\mathbf{1 1 0 1}$ & $\mathbf{2 3 4}$ & $\mathbf{1 7 3}$ & $\mathbf{1 3 4}$ \\
RMSE & & $\mathbf{2 9 4 . 4 4}$ & $\mathbf{2 3 0 . 4}$ & $\mathbf{3 3 . 1 8}$ & $\mathbf{1 5 . 3}$ & $\mathbf{1 3 . 1 5}$ & $\mathbf{4 . 2 4}$ \\
\hline
\end{tabular}




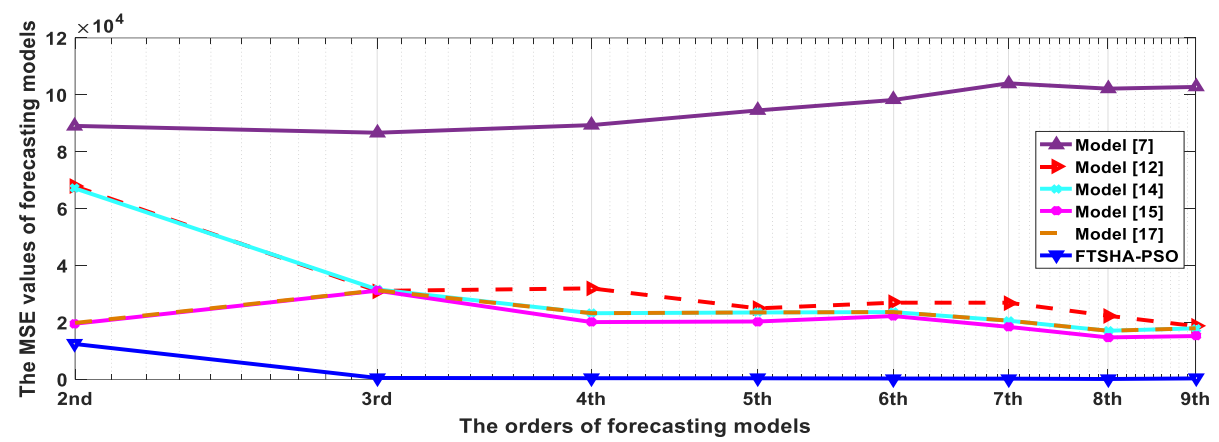

Figure 4 The curves present the MSE values between the FTSHA-PSO model and its counterparts based on the various high-order FTS.

To be easily visualized, Figure 4 depicts the trend in term of forecasting accuracy between the FTSHA-PSO model and its counterparts with different high-order FLRs. From these curves, it can be seen that forecasting accuracy of the FTSHA-PSO model is more precise than those of compared models under different high-order FLRs at all. To sum up, demonstrations above show that the FTSHA-PSO model outperform the existing models based on high-order FTS model with different number of intervals in forecasting the enrolments of University of Alabama.

\section{Experimental results in the testing phase}

Based on the historical enrolments data for the past years, we can forecast the new enrollment for the next year only. For example, the historical data of enrollments from year 1971 to 1989, is utilized to forecast the new enrollment of year 1990. Similarly, a new enrolment of year 1991 can be forecasted based on the enrollments from years 1971 - 1990. After the training data have been well trained by the FTSHA-PSO, the future enrollment values could be accomplished to compare with the ones of the forecasting models presented in articles [4,11,14,37]. The comparison of the forecasted results produced based on the $3^{\text {rd }}$-order FLR the number of different intervals and the highest vote $W_{h}=20$ (constantdefined by the user) in Tables 16 and 17. From Tables 16 and 17, it can be seen that the FTSHA-PSO model obtains the lowest RMSEs value of 98.6 and 72.53 among 5 compared models, respectively. From these results indicate that the our FTSHA-PSO model is more precise than its counterparts based on $3^{\text {rd }}$ order FTS with different number of intervals.

Table 16 A comparison of the forecasting results between the FTSHA-PSO model and other models with the number of intervals $=7$ and which use vote $W_{h}=20$.

\begin{tabular}{cccccccc}
\hline \multirow{2}{*}{ Year } & \multirow{2}{*}{ Real data } & \multirow{2}{*}[\mathbf{4}]{} & \multirow{2}{*}[\mathbf{11}]{} & {$[\mathbf{1 4}]$} & $\begin{array}{c}\text { ATVF-KM } \\
{[\mathbf{3 7 ]}}\end{array}$ & $\begin{array}{c}\text { ATVF-PSO } \\
{[\text { [37] }}\end{array}$ & \multirow{2}{*}{ FTSHA-PSO } \\
\hline 1990 & 19328 & 18168 & 18059 & 18326 & 19525 & 19226 & 19308 \\
1991 & 19337 & 18909 & 18669 & 19212 & 19150 & 19182 & 19351.9 \\
1992 & 18876 & 19609 & 19083 & 19203 & 18933 & 18876 & 19045 \\
RMSE & & $\mathbf{7 7 3 . 6 6}$ & $\mathbf{5 7 6 . 6 6}$ & $\mathbf{4 8 4 . 1 6}$ & $\mathbf{1 6 0 . 4 3}$ & $\mathbf{1 0 7 . 2 9}$ & $\mathbf{9 8 . 6}$ \\
\hline
\end{tabular}

Table 17 A comparison of the forecasting results between the FTSHA-PSO model and other models with the number of intervals $=14$ and which use vote $W_{h}=20$.

\begin{tabular}{|c|c|c|c|c|c|c|c|}
\hline Year & Real data & [4] & [11] & {$[14]$} & $\begin{array}{c}\text { ATVF-KM } \\
\text { [37] }\end{array}$ & $\begin{array}{c}\text { ATVF-PSO } \\
{[37]}\end{array}$ & FTSHA-PSO \\
\hline 1990 & 19328 & 18162 & 17862 & 18120 & 19287 & 19238 & 19230 \\
\hline 1991 & 19337 & 18721 & 18633 & 19027 & 18811 & 19224 & 19336.74 \\
\hline 1992 & 18876 & 19221 & 19085 & 19137 & 18836 & 19224 & 18954.6 \\
\hline RMSE & & 709 & 653.66 & 621.91 & 305.7 & 92.35 & 72.53 \\
\hline
\end{tabular}




\section{Conclusions}

In this paper, we propose a hybrid FTS forecasting model combining HA and PSO, namely FTSHAPSO. The FTSHA-PSO model has addressed 2 issues are considered to be important and greatly affect the forecasting accuracy that is the problem about determining of length of intervals and how to establish fuzzy relationship groups. To overcome the limitations of fuzzy time series models using the fuzzy relationship groups, the FTSHA-PSO model uses the concept of time variant fuzzy relationship group to calculate the forecasting results output. Using this fuzzy relationship group has proved to be more appropriate for practical use. In addition, the PSO optimization technique is applied in finding the optimal lengths of intervals from the universe of discourse to improve the forecast accuracy of the FTSHA-PSO model. Among mining techniques and finding the best solution, PSO is considered to perform better compared to other heuristic techniques in terms of success rate and solution quality. By combining HA and PSO technique, the forecasting efficiency of the FTSHA-PSO model can be significantly improved. The experimental results on dataset of University of Alabama show that, in many cases, the FTSHA-PSO model gets better forecasting performance than the existing ones. Details of the comparison are shown in Tables 12 17. Although our FTSHA-PSO model shows that the superior forecasting capability compared with existing forecasting models based on the high-order FLRs. However, determining FLR in high-order is more complicated and computationally more expensive than $1^{\text {st }}$-order. Therefore, development of new approaches that can automatically find the optimal order of the high-order FLRs is a worthy idea in FTS forecasting model. Those will be the future work closely related to this research.

\section{Acknowledgment}

This work was supported by Thai Nguyen University of Technology (TNUT).

\section{References}

[1] LA Zadeh. Fuzzy sets. Inf. Control 1965, 8, 338-53.

[2] Q Song and BS Chissom. Fuzzy time series and its models. Fuzzy Sets Syst. 1993; 54, 269-77.

[3] Q Song and BS Chissom. Forecasting enrollments with fuzzy time series - Part I. Fuzzy Sets Syst. 1993; 54, 1-9.

[4] SM Chen. Forecasting enrollments based on fuzzy time series. Fuzzy Sets Syst. 1996; 81, 311-9.

[5] HK Yu. Weighted fuzzy time series models for TAIEX forecasting. Physica A 2005; 349, 609-24.

[6] VR Uslu, E Bas, U Yolcu and E Egrioglu. A fuzzy time series approach based on weights determined by the number of recurrences of fuzzy relations. Swarm Evol. Comput. 2014; 15, 19-26.

[7] SM Chen. Forecasting enrollments based on hight-order fuzzy time series. Cybern. Syst. 2002; 33, $1-16$.

[8] K Huarng. Effective lengths of intervals to improve forecasting in fuzzy time series. Fuzzy Sets Syst. $2001 ; 123,387-94$.

[9] LW Lee, LH Wang, SM Chen and YH Leu. Handling forecasting problems based on two-factors high-order fuzzy time series. IEEE Trans. Fuzzy Syst. 2006; 14, 468-77.

[10] SM Chen and K Tanuwijaya. Fuzzy forecasting based on high- order fuzzy logical relationships and automatic clustering techniques. Expert Syst. Appl. 2011; 38, 15425-37.

[11] SM Chen and NY Chung. Forecasting enrollments of students by using fuzzy time series and genetic algorithms. Int. J. Inf. Manag. Sci. 2006; 17, 1-17.

[12] SM Chen and NY Chung. Forecasting enrollments using high-order fuzzy time series and genetic algorithms. Int. Intell. Syst. 2006; 21, 485-501.

[13] LW Lee, LH Wang and SM Chen. Temperature prediction and TAIFEX forecasting based on hight order fuzzy logical ralationship and genetic simulated annealing techniques. Expert Syst. Appl. 2008; 34, 328-36.

[14] IH Kuo, SJ Horng, TW Kao, TL Lin, CL Lee and Y Pan. An improved method for forecasting enrollments based on fuzzy time series and particle swarm optimization. Expert Syst. Appl. 2009; 36, 6108-17.

[15] YL Huang, SJ Horng, M He, P Fan, TW Kao, MK Khan, JL Lai and IH Kuo. A hybrid forecasting model for enrollments based on aggregated fuzzy time series and particle swarm optimization. Expert Syst. Appl. 2001; 38, 8014-23.

[16] LY Hsu, SJ Horng, TW Kao, YH Chen, RS Run, RJ Chen, JL Lai and IH Kuo. Temperature prediction and TAIFEX forecasting based on fuzzy relationships and MTPSO techniques. Expert Syst. Appl. 2010; 37, 2756-70. 
[17] NC Dieu and NV Tinh. Fuzzy time series forecasting based on time-depending fuzzy relationship groups and particle swarm optimization. In: Proceedings of the $9^{\text {th }}$ National Conference on Fundamental and Applied Information Technology Research, Can Tho, Vietnam. 2016, p. 125-33.

[18] JI Park, DJ Lee, CK Song and MG Chun. TAIFEX and KOSPI 200 forecasting based on two-factors high-order fuzzy time series and particle swarm optimization. Expert Syst. Appl. 2010; 37, 959-67.

[19] SM Chen and BHP Dang. Fuzzy time series forecasting based on optimal partitions of intervals and optimal weighting vectors. Knowl. Based Syst. 2016; 118, 204-16.

[20] SM Chen and WS Jian. Fuzzy forecasting based on two-factors second-order fuzzy-trend logical relationship groups, similarity measures and PSO techniques. Inf. Sci. 2017; 391-392, 65-79.

[21] M Bose and K Mali. A novel data partitioning and rule selection technique for modelling high-order fuzzy time series. Appl. Soft Comput. 2017; 63, 87-96.

[22] Z Tian, P Wang and T He. Fuzzy time series based on K-means and particle swarm optimization algorithm. In: Proceedings of the International Conference on Man-Machine-Environment System Engineering, Xi'an, China. 2016, p. 181-9.

[23] Z Zhang and Q Zhu. Fuzzy time series forecasting based on k-means clustering. Open J. Appl. Sci. $2012 ; 2,100-3$.

[24] NV Tinh and NC Dieu. Improving the forecasted accuracy of model based on fuzzy time series and k-means clustering. J. Sci. Technol. 2017; 3, 46-55.

[25] E Bulut, O Duru and S Yoshida. A fuzzy time series forecasting model formulti-variate forecasting analysis with fuzzy c-means clustering. World Acad. Sci. Eng. Technol. 2012; 63, 765-71.

[26] S Askari and N Montazerin. A high-order multi-variable Fuzzy Time Series forecasting algorithm based on fuzzy clustering. Expert Syst. Appl. 2015; 42, 2121-35.

[27] NC Ho, NC Dieu and VN Lan. The application of hedge algebras in fuzzy time series forecasting. $J$. Sci. Technol. 2016; 54, 161-77.

[28] NC Ho and W Wechler. Hedge algebra: An algebraic approach to structures of sets of linguistic truth values. Fuzzy Sets Syst. 1990; 35, 281-93.

[29] H Tung, ND Thuan and VM Loc. The partitioning method based on hedge algebras for fuzzy time series forecasting. J. Sci. Technol. 2016; 54, 571-83.

[30] NV Tinh. Enhanced forecasting accuracy of fuzzy time series model based on combined fuzzy Cmean clustering with particle swam optimization. Int. J. Comput. Intell. Appl. 2020; 19, 2050017.

[31] J Kennedy and R Eberhart. Particle swarm optimization. In: Proceedings of the IEEE International Conference on Neural Network, Perth, WA, Australia. 1995, p. 1942-8.

[32] NC Ho, TT Son and DT Long. Hedge algebras with limited number of hedges and applied to fuzzy classification problems. Vietnam J. Sci. Technol. 2012; 48, 13-22.

[33] L Wang, XD Liu and W Pedrycz. Effective intervals determined by information granules to improve forecasting in fuzzy time series. Expert Syst. Appl. 2013; 40, 5673-79.

[34] L Wang, X Liu, W Pedrycz and Y Shao. Determination of temporal information granules to improve forecasting in fuzzy time series. Expert Syst. Appl. 2014; 41, 3134-42.

[35] W Lu, X Chen, W Pedrycz, X Liu and J Yang. Using interval information granules to improve forecasting in fuzzy time series. Int. J. Approx. Reason. 2015; 57, 1-18.

[36] Y Wang, Y Lei, X Fan and Y Wang. Intuitionistic fuzzy time series forecasting model based on intuitionistic fuzzy reasoning. Math. Probl. Eng. 2016; 2016, 5035160.

[37] K Khiabani and SR Aghabozorgi. Adaptive time-variant model optimization for fuzzy-time-series forecasting. IAENG Int. J. Comput. Sci. 2015; 42, 107-16. 